\title{
Optimal Passive Tracking of Ground Targets
}

\author{
Stuart Kramer, $\mathrm{PhD}$ \\ Department of Aeronautics and Astronautics \\ Air Force Institute of Technology \\ Wright-Patterson AFB, OH 45432
}

\begin{abstract}
Passive tracking of ground targets from airborne vehicles continues to be an important estimation problem. Traditional point estimators such as extended Kalman filters have had limited success. It is not clear, however, whether this is due to the limitations of the estimators faced with a nonlinear problem, or due to inherent limitations in the situation itself. In order to explore this issue, a near-optimal estimate must be available. For this study, the optimal estimate was computed using a procedure that numerically computes the posterior location probability density after each measurement. Although not computationally feasible for real-time applications, it does provide an accurate benchmark. Preliminary results indicate that the optimal estimate is a moderate improvement over the EKF, both in quality of the estimate and consistency between the predicted and actual estimate error variance. This indicates that some improvement is possible in point estimators for this problem.
\end{abstract}

\section{Introduction}

The passive tracking problem is encountered whenever one is attempting to locate an emitting target by observing only the direction from which the emission arrives. The name comes from the fact this information can be obtained passively, without active emission on the receiver's part. This is clearly an advantage in a military application, since the observer need not advertise its position. The major drawbacks with this approach are 1) that such observations rarely include any distance information, and 2) the observations depend nonlinearly on the target location. The result is a potentially unobservable, highly nonlinear estimation problem.

Previous attacks on this problem have centered around the use of point estimators, most based on some variation of the extended Kalman filter (EKF). These variations are generally either different definitions of the state variables (eg, [1]), or modifications of the basic EKF (eg, [2]).
So-called Bayesian or density based estimators take a broader view and aim to compute at least a close approximation of the conditional posterior density of the unknown quantities [3]. With the probability density function in hand, the true optimal estimate can be obtained for any given loss function. Generally, the minimum variance estimate is the desired one, and since that is what the EKF is intended to produce, that is what we will use in this study. The difficulty with this approach is that the required densities are not usually calculable in closed form and numerical approximations must be used [4]. This usually results in estimators that are not appropriate for real-time use. On the other hand, this does provide a useful benchmark for other estimators, as well as an alternative viewpoint that can help illuminate the behavior of those estimators.

As will be shown, the approximate Bayes' estimate when compared to the EKF is generally superior, both in terms of lower average error, and in terms of consistency between the estimator's predicted and observed error variance. Viewing the EKF from the Bayesian perspective provides some clues to its relatively poor performance.

\section{Problem Description}

For this study, we will consider a simplified problem suggested by Bar-Shalom [5] and depicted in Figure 1. The receiver platform flies at a constant nominal altitude and forward speed, and takes corrupted directional measurements of the target emitter on the ground. The target is constrained to travel on the level ground along the line of flight of the receiver with random position and velocity perturbations. The time history of the system as a function of time $k$ can be described by the following discrete time model:

$$
\begin{gathered}
p_{x, k}=\bar{p}_{x, k}+\delta_{x, k} ; \bar{p}_{x, k}=p_{v} * k \\
p_{y, k}=\bar{p}_{y, k}+\delta_{y, k} ; \bar{p}_{y, k}=p_{h} \\
x_{k+1}=\left[\begin{array}{c}
t_{x, k+1} \\
t_{v, k+1}
\end{array}\right]=\left[\begin{array}{cc}
1 & 1 \\
0 & 1
\end{array}\right] x_{k}+w_{k} \\
z_{k}=\tan ^{-1}\left(\frac{-p_{y, k}}{t_{x, k}-p_{x, k}}\right)+r_{k}=h\left(p_{x, k}, p_{y, k}, x_{(1), k}\right)
\end{gathered}
$$




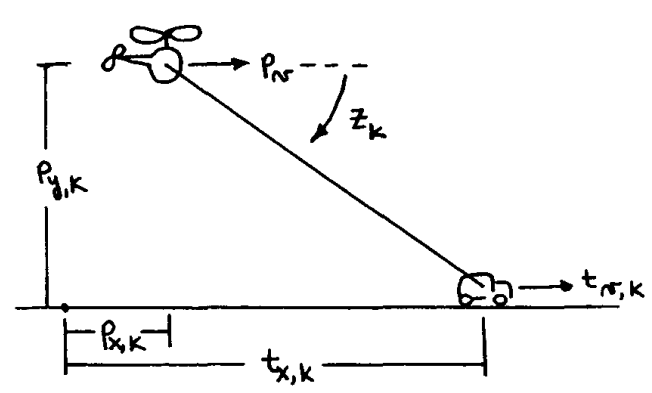

Figure 1 - Problem Geometry

The scalar noises $\delta_{x, k}, \delta_{y, k}$, and $r_{k}$ are independent white zero-mean Gaussian processes with variances $q_{x}, q_{y}$, and $q_{r}$ respectively. The target state noise $w_{k}$ is white zero-mean Gaussian with covariance matrix $R$. The nominal receiver platform position $(\bar{p})$ is assumed known, as are the noise variances.

With this formulation, the objective is to calculate an estimate of the target position and velocity $\left(x_{k}\right)$ using successive measurements of the bearing angle to the target $\left(z_{k}\right)$. By restricting the problem in this way, it is clearly observable, yet retains the complex nonlinearities in the observation relation. In addition, this model reflects the realistic effect of uncertainty in the receiver location.

In evaluating the performance of the estimator, we are concerned about the ability of the estimator to give "good" estimates of both the state and the error in that state estimate. The effectiveness of the state estimator can be gauged qualitatively by observing the rate of convergence (or lack thereof) of the estimates, and the eventual average steady state error. For this, the mean squared error is a useful measure. Measuring consistency (in this case the degree to which the actual error variance and the filter predicted variance agree) we can be more quantitative. Assuming that the estimates at time $i, \hat{x}_{i}$, are distributed Gaussian with variance $\sigma_{i}^{2}$ as predicted by the filter, the sum of the normalized errors squared for $n$ independent trials, $\sum_{j=1}^{n}\left(x_{i, j}-\hat{x}_{i, j}\right)^{2} / \sigma_{i, j}^{2}$ will be distributed $\chi_{n-1}^{2}$. This can be used to form the basis of a test of hypothesis regarding the actual error distribution [6].

\section{EKF Implementation}

The EKF for this problem is mostly a straightforward application of the basic equations which can be found in a number of sources (eg, [7]). The only minor complication is the need to modify the observation noise covariance calculation to include the effects of receiver position uncertainty. Taking the first order terms from a series expansion of the observation function (1) about the nominal observer position, we get

$$
z_{k}=h\left(\bar{p}_{x, k}, \bar{p}_{y, k}, x_{(1), k}\right)+\frac{\partial h}{\partial p_{x}} \delta_{x, k}+\frac{\partial h}{\partial p_{y}} \delta_{y, k}+r_{k}
$$

Taking the last three terms as an equivalent noise $w_{k}$,

$$
\mathrm{E}\left[w_{k}\right]=0 ; \mathrm{E}\left[w_{k}^{2}\right]=\frac{\bar{p}_{y, k}^{2} q_{x}+\left(x_{(1), k}-\bar{p}_{x, k}\right)^{2} q_{y}}{\left(\bar{p}_{y, k}^{2}+\left(x_{(1), k}-\bar{p}_{x, k}\right)^{2}\right)^{2}}+q_{r}
$$

The equivalent noise is then used in the formulation of the EKF.

The EKF is initialized based on the first observation. The initial position estimate is given by

$$
\hat{x}_{(1), 0}=\bar{p}_{x, 0}-\frac{\bar{p}_{y, 0}}{\tan z_{0}}
$$

The variance of the initial estimate is computed using the first order terms of the series expansion of (4) about the nominal platform position and initial observation. The initial velocity estimate is 0 with variance 1 , uncorrelated with the initial position estimate.

The performance of the EKF in this case is not particularly bad. The state estimates converge in a generally well-behaved way, and the filter shows no signs of covariance collapse or divergence. On the other hand, it is not clear that it is very good either. In particular, the observed variance of the estimates is not consistent with the EKF generated variance estimate, as will be shown in the numerical results section. Bar-Shalom reports that neither adding second order terms nor performing local iterations (ie, using an iterated EKF) improves the estimator performance. Some improvement can be obtained by tuning the filter by increasing the noise variances used in the EKF. This is not very satisfying since it is rather ad hoc and sensitive to the actual system state. Therefore it is necessary to know the true optimal estimate in order to accurately assess this estimator's performance and indicate whether it may be worth looking for better options.

\section{Optimal (Bayes') Estimator}

The optimal estimator is based on actually computing the probability density function for the system state conditioned on the observations. With some minor restrictions on the noises, the recursion can be formally written as

$$
\begin{gathered}
p\left(x_{0} \mid z_{0}\right)=p_{0} \\
p\left(x_{k} \mid z_{0}, \ldots, z_{k}\right)=C p\left(x_{k} \mid z_{0}, \ldots, z_{k-1}\right) p\left(z_{k} \mid x_{k}, z_{0}, \ldots, z_{k-1}\right) \\
p\left(x_{k+1} \mid z_{0}, \ldots, z_{k}\right)= \\
\int p\left(x_{k} \mid z_{0}, \ldots, z_{k}\right) p\left(x_{k+1} \mid x_{k}, z_{0}, \ldots, z_{k}\right) d x_{k}
\end{gathered}
$$

where the measurement update density $p\left(z_{k} \mid x_{k}, z_{0}, ., z_{k-1}\right)$ and the time update density $p\left(x_{k+1} \mid x_{k}, z_{0}, ., z_{k}\right)$ are determined by the system model and the noise densities.

As noted above, however, one must generally resort to numerical approximations solve the recursion. For this study, the piece-wise constant density approximation de- 


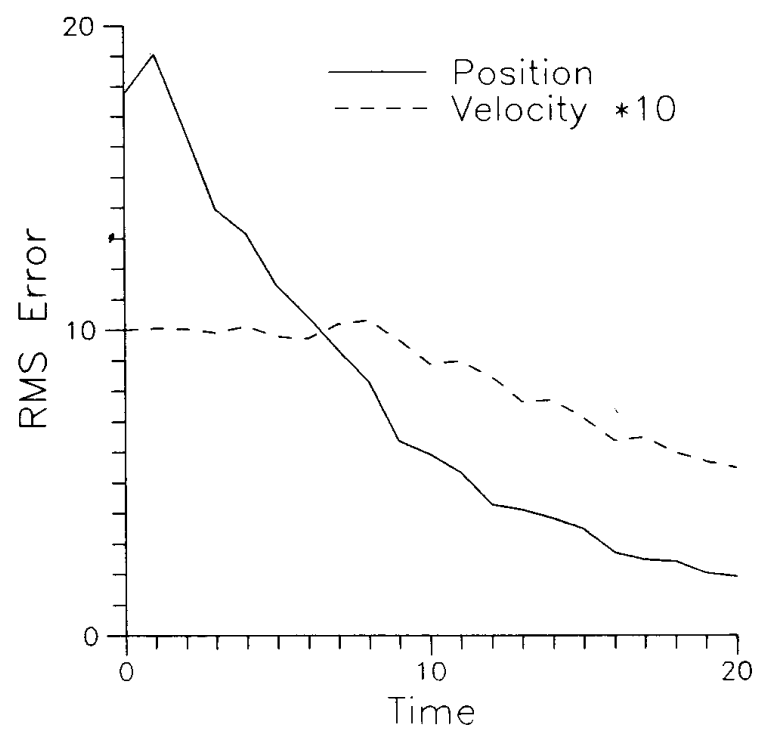

Figure 2 - EKF RMS Errors

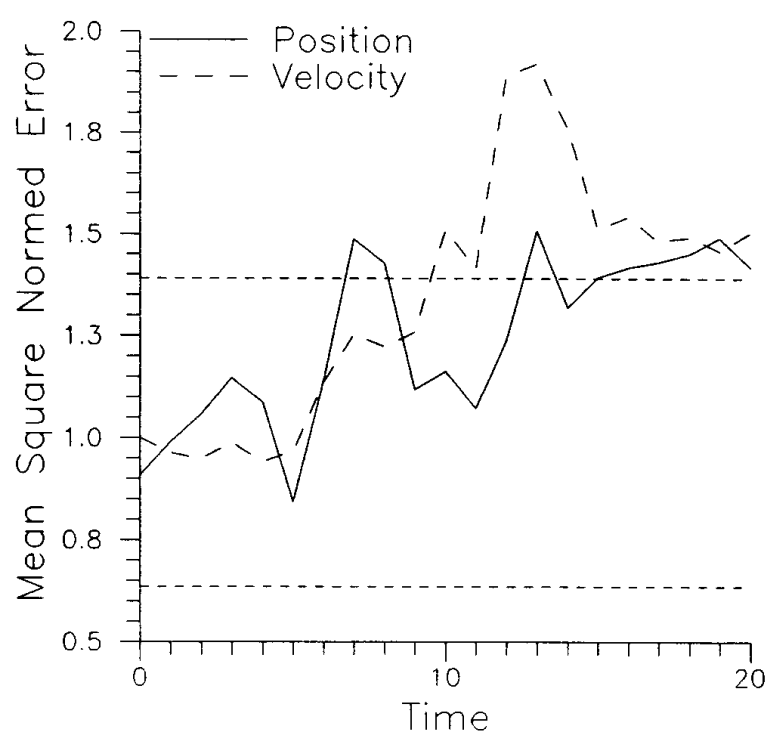

Figure 3 - EKF Mean Squared Normalized Errors

veloped by Kramer and Sorenson was used [8]. In this approach, the relevant densities are approximated with piecewise constant functions over their support. This yields a simplified recursion for updating the state probability density.

\section{Numerical Results}

Both estimators were evaluated via Monte Carlo simulation so that both used the same sequences of observa-

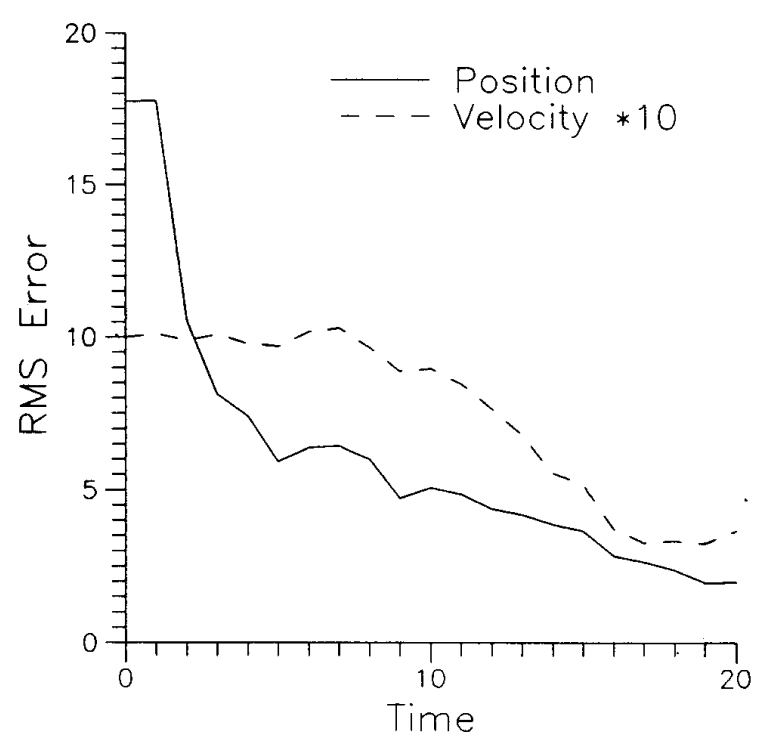

Figure 4 - Bayes' RMS Errors

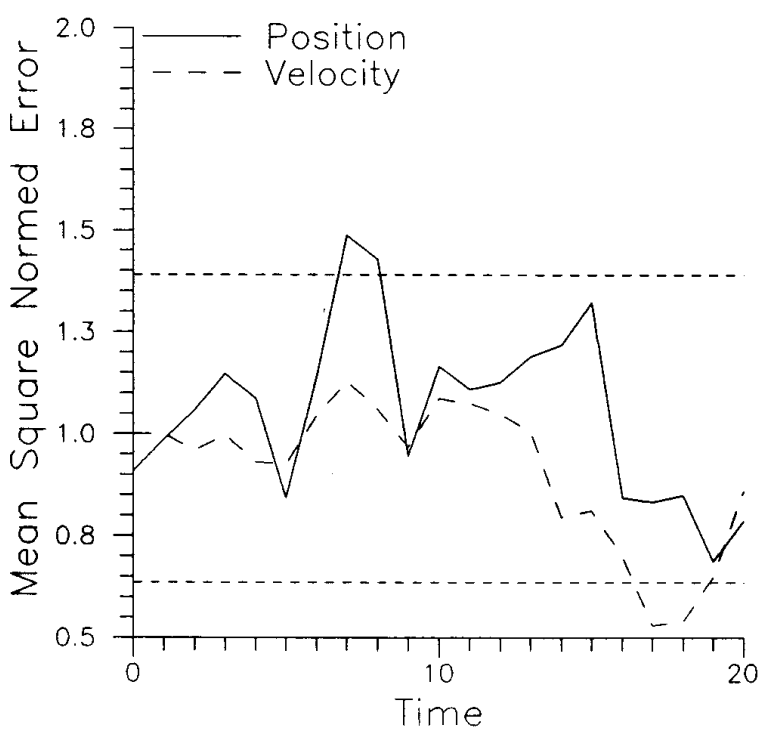

Figure 5 - Bayes' Mean Squared Normalized Errors

tions. The particular system parameter values were chosen arbitrarily as follows:

$$
\begin{gathered}
x_{0}=\left[\begin{array}{c}
80 \\
1
\end{array}\right] \\
q_{x}=q_{y}=1.0 ; q_{r}=\left(3^{\circ}\right)^{2} \\
R=\left[\begin{array}{cc}
0.0025 & 0.005 \\
0.005 & 0.01
\end{array}\right]
\end{gathered}
$$


Figure 2 shows the RMS error in the two states for the EKF evaluated over 51 independent repetitions. As noted above, the state estimates converge reasonably. Figure 3 shows the mean squared normalized errors for the same series of runs. At the $95 \%$ confidence level, the values should be between 1.4 and 0.64 . Since the values are consistently outside the limits over the latter half of the interval, we conclude the estimator is inconsistent.

Figures 4 and 5 show the same measures for the Bayes' estimator. Comparing with the EKF, the state estimates converge more rapidly. Similarly, the mean squared normalized errors are generally less, particularly in the second part of the interval. Note that the EKF tends to underestimate the error variance while the Bayes' slightly overestimates it.

\section{Interpretation from Bayesian Viewpoint}

By identifying reasons for the EKF's poorer performance, we may find potential improvements. Looking at the EKF from a Bayesian viewpoint can help here. The EKF can be interpreted as an approximate implementation of the density recursion (5) where the initial state density and the time and measurement update densities are Gaussian approximations to the true densities. Since the system (target) dynamics are linear and the input noise is Gaussian, the time update density is in fact Gaussian. The measurement density however is not. On initial inspection, this does not seem to be a significant problem, since the approximate variance (3) is quite good for the range of parameters of interest. The difficulty is that the variance for the Gaussian approximation must be calculated at the current estimated target state. This leads to increasing mismatch between the EKF measurement density and the true density in the tails. This is illustrated in Figure 6, which shows the two densities at $k=10$. This skewness also indicates that including second order terms would not improve the the EKF significantly since they would still be symmetric. Similarly, since the Gaussian is a good approximation near the center, local iterations would probably have little effect.

This also indicates that the true error density is not Gaussian. Figure 7 shows a representative Bayes' calculated error density for $k=5$. It is not clear whether this is sufficiently non-Gaussian to seriously affect the $\chi^{2}$ test used above.

Finally, this implies that the EKF may exhibit a bias because of the skewness of the underlying densities. This was not explored in this study and remains for future research.

\section{Conclusions}

This study briefly explored the performance of the EKF as a state estimator in a restricted passive tracking problem. The performance of the EKF was compared to a approximate optimal minimum variance estimate. The EKF

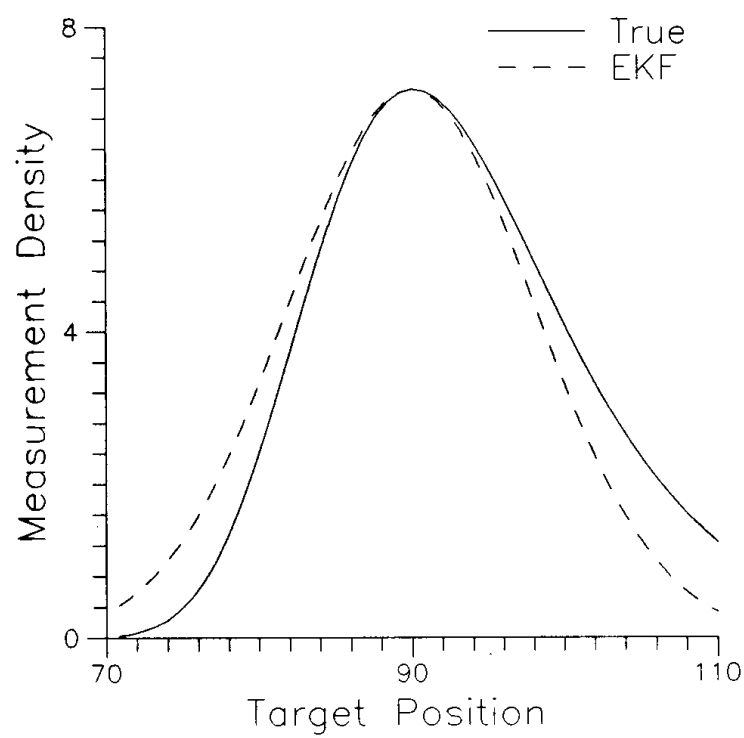

Figure 6 - EKF vs Bayes' Messurement Density

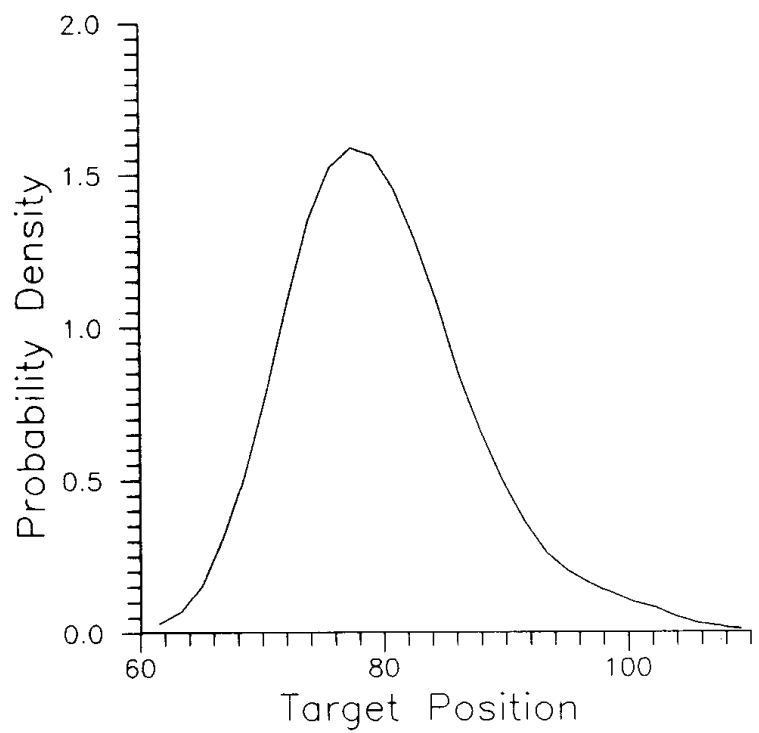

Figure 7 - Bayes' Calculated Position Error Density

was found to have poorer state estimate convergence, and poorer agreement between the estimator predicted error variance and the actual error variance. Viewing the EKF as another approximate Bayes' estimator pointed out that the EKF deficiencies are partly a result of the mismatch between the EKF assumption of Gaussian update densities and the true non-Gaussian measurement update density. The next step is to use this insight to modify the EKF to provide closer to optimal performance. 


\section{References}

[1] V J Aidala, S E Hammel, "Utilization of modified polar coordinates for bearings-only tracking," IEEE Trans Auto Contr, AC-28, 283-294, 1983.

[2] H Weiss, J B Moore, "Improved extended Kalman filter design for passive tracking," IEEE Trans Auto Contr, AC-25, 807-811, 1980.

[3] V Peterka, "Bayesian system identification," Automat. ica, 17, 41-53, 1981.

[4] H W Sorenson, "On the development of practical nonlinear filters," Information Sciences, 7, 253-270, 1974.

[5] Y Bar-Shalom, private correspondence, 1987.

[6] W Mendenhall, R L Scheaffer, Mathematical statistics with applications. North Scituate, Mass: Duxbury Press, 1972.

[7] B D O Anderson, J B Moore, Optimal filtering. Englewood Cliffs, NJ: Prentice-Hall, 1979.

[8] S C Kramer, H W Sorenson, "Recursive Bayesian estimation using piece-wise constant approximations," $A u$ tomatica, 24, 789-801, 1988. 